\title{
Extensions of the Justen-Ramlau blind deconvolution method
}

\author{
Tristan A. Hearn* Lothar Reichel ${ }^{\dagger}$
}

\begin{abstract}
Blind deconvolution problems arise in many image restoration applications. Most available blind deconvolution methods are iterative. Recently, Justen and Ramlau proposed a novel non-iterative blind deconvolution method. The method was derived under the assumption of periodic boundary conditions. These boundary conditions may introduce oscillatory artifacts into the computed restoration. We describe extensions of the Justen-Ramlau method that allow the use of Neumann and antireflective boundary conditions.
\end{abstract}

\section{Introduction}

The blurring of a digital image is often modeled by a linear convolution operation,

$$
g(x, y)=(k * f)(x, y)=\iint_{\Omega} k(x-\psi, y-\xi) f(\psi, \xi) d \psi d \xi, \quad(x, y) \in \Omega,
$$

where $f$ represents the exact image, $g$ is the resulting blurred image, $k$ is the kernel function of the blurring operator, and $\Omega$ is a rectangular region in $\mathbb{R}^{2}$. In the context of digital image restoration, the function $k$ is often referred to as a point spread function (PSF). Thus, the problem of determining $f$, given $g$ and $k$, can be expressed as a Fredholm integral equation of the first kind

$$
g=K f .
$$

The solution of equation (2) for $f$, given $g$ and $K$, is referred to as deconvolution. In applications of interest, the kernel $k$ is a smooth function. Then solving (2) is an ill-posed problem in the sense of Hadamard due to the lack of continuous dependence of the solution $f$ on the data $g$. The computation of a meaningful approximate solution of (2) in finite-precision arithmetic is generally only possible with the aid of regularization methods, such as truncated singular value decomposition or Tikhonov regularization. These methods replace the ill-posed problem (2) by a nearby problem, whose solution is less sensitive to perturbations introduced by discretization and computations in finite precision.

*NASA Glenn Research Center, 21000 Brookpark Road, Cleveland, OH 44135, USA, and Department of Mathematical Sciences, Kent State University, Kent, OH 44242, USA. E-mail: tristan.a.hearn@nasa.gov

${ }^{\dagger}$ Department of Mathematical Sciences, Kent State University, Kent, OH 44242, USA. E-mail: reichel@math.kent.edu. Research supported in part by NSF grant DMS-1115385. 
In the case when the point spread function $k$ is not known, the numerical solution of (2) is referred to as blind deconvolution. Due to the lack of unicity of the computed solution $f$, blind deconvolution also is an ill-posed problem. Blind deconvolution problems arise in many applications in science and engineering, including astronomical, biological, and medical imaging; see, e.g., [3] for recent discussions of methods and applications.

Most of the available blind deconvolution methods are iterative; see, e.g., $[2$, $3,5,6,7,10,12,14,15,16,18,19,21]$ and references therein. Recently, Justen and Ramlau [13] introduced a novel non-iterative method. This method can be applied when the PSF $k$ satisfies certain smoothness and symmetry conditions. Justen and Ramlau [13] considered the situation when the PSF $k$ and the data $g$ are periodic. Advantages of the non-iterative method by Justen and Ramlau [13], in comparison with available iterative methods for blind deconvolution, include its simplicity, which makes implementation fairly straightforward, and its speed. However, the use of periodic boundary conditions (BCs) for the PSF $k$ and the data $g$ may lead to oscillatory artifacts propagating from the boundaries of the restored image. These artifacts can be avoided or attenuated by using other BCs. For instance, reflective (or "Neumann") BCs impose continuity of the computed solution across the boundary of the image. Neumann boundary conditions suppress many oscillatory artifacts in the computed solution that may be present when periodic boundary conditions are imposed. This paper describes extensions of the JustenRamlau method to reflective and antireflective BCs.

The main contribution of this paper is to present a simple blind deconvolution method that yields approximations of the original image and of the PSF with fairly little computational effort. The method in many situations gives better approximations than the scheme by Justen and Ramlau [13]. This is illustrated in Section 5. It may in some applications be attractive to use the computed image and PSF as initial approximations for one of the available iterative methods to enhance the quality of the computed image further. Having a fairly accurate initial approximation for the iterative methods reduces the computational effort required by the latter and may also affect the quality of the computed image; see Carasso [4] for a discussion on a particular post-processing method. We are presently investigating this kind of hybrid methods.

In applications that require image deblurring, the available contaminated image $g$ generally also is corrupted by noise $\eta$, which may stem from imperfections in the recording of $g$ and from storage in finite-precision. The exact image $f$ then satisfies the equation

$$
\breve{g}=K f+\eta .
$$

We consider this situation in Section 5, which presents computed examples.

This paper is organized as follows. In Section 2, the convolution operation and the effects of the boundary conditions on the solution of the associated inverse problem are discussed in terms of finite-dimensional linear algebra. Section 3 describes the non-iterative blind deconvolution method of Justen and Ramlau. The periodic BCs and shift invariance used in this method allow the application of the Fourier transform to simplify the problem. In Section 4, a modified algorithm that admits more general BCs, including Neumann and antireflective BCs, is derived. In particular, a novel decomposition of the blurring matrix that allows efficient computations 
is proposed. Numerical examples determined with the modified method are provided in Section 5 using periodic, reflective and antireflective boundary conditions. Section 6 contains concluding remarks.

\section{The Linear Algebra of Image Deblurring}

Consider a discretized restatement of the deconvolution problem, where the functions $f, k$, and $g$ are represented, respectively, by the finite dimensional matrices $F, K, G \in \mathbb{R}^{m \times n}$. Each entry of $F$ and $G$ represents a pixel in a gray-scale digital image. The numerical value of each pixel is called the intensity, which determines the brightness of a small portion of $f$ and $g$ in $\Omega$. Similarly, the entries of $K$ repre-

sent discrete samplings of the convolution kernel $k$ in $\Omega$. In this setting, the blurring operation (1) takes the form

$$
G_{i, j}=\sum_{h=1}^{n} \sum_{l=1}^{m} K_{i-h, j-l} F_{h, l},
$$

involving the matrices $K, F, G \in \mathbb{R}^{m \times n}$. Next we introduce two operators that allow us to represent matrices as vectors, and vectors as matrices.

Definition 1. Let $A=\left[a_{i j}\right] \in \mathbb{R}^{m \times n}$. Then the vectorization operator

$$
\text { vec }: \mathbb{R}^{m \times n} \rightarrow \mathbb{R}^{m n}
$$

applied to $A$ yields the column vector formed by successively stacking the columns $a_{j}$ of $A$. That is

$$
\operatorname{vec}(A)=\left[\begin{array}{c}
a_{1} \\
a_{2} \\
\vdots \\
a_{n}
\end{array}\right]=\left[\begin{array}{c}
a_{11} \\
\vdots \\
a_{m 1} \\
\vdots \\
a_{m n}
\end{array}\right] .
$$

Definition 2. Let $x=\left[x_{i}\right] \in \mathbb{R}^{m n}$. Then the matrixization operator

$$
\text { mat }: \mathbb{R}^{m n} \rightarrow \mathbb{R}^{m \times n}
$$

applied to $x$ yields the matrix formed by successive column-wise filling of an $m \times n$ matrix with the elements of $x$, i.e.,

$$
\operatorname{mat}(x)=\left[\begin{array}{cccc}
x_{1} & x_{m+1} & \cdots & x_{m(n-1)} \\
\vdots & \vdots & & \vdots \\
x_{m} & x_{2 m} & \cdots & x_{m n}
\end{array}\right] \text {. }
$$

The vec and mat operators may be executed in MATLAB using the reshape command. It follows from Definitions 1 and 2 that for any $A \in \mathbb{R}^{m \times n}$ and $x \in \mathbb{R}^{m n}$

$$
\operatorname{mat}(\operatorname{vec}(A))=A \text { and } \operatorname{vec}(\operatorname{mat}(x))=x .
$$

The purpose of introducing the vectorization operator is to allow for (4) to be written as a linear system of equations

$$
A x=b,
$$


where $x=\operatorname{vec}(F) \in \mathbb{R}^{m n}, b=\operatorname{vec}(G) \in \mathbb{R}^{m n}$, and $A \in \mathbb{R}^{m n \times m n}$ is a matrix which encodes the discrete convolution operation.

Since it is assumed that $F, G$, and $K$ are matrices of the same dimensions, $\mathrm{BCs}$ for the convolution operation must be prescribed, i.e., we have to prescribe the contribution to the blurred image from pixels outside of the field of view of the recorded image. Figure 1 shows extensions of a sample image assuming zero-padded, periodic, reflective, and antireflective BCs. The exact assumptions underlying each boundary condition are as follows:

(i) Zero-padded BCs, also referred to as Dirichlet BCs: All pixels outside of the field of view of the recorded image are assumed to have the value zero. Zeropadded boundary conditions are perhaps most useful in a situation in which it may be assumed that most of the data has a numerical value near zero such as in many astronomical images. This results in a blurring matrix with a Toeplitz structure, but generally cannot be structurally characterized any further. Consequently, image deblurring problems involving zero-padded BCs do not involve blurring operators which are quickly diagonalizable using fast transforms on the components of the recorded image and PSF matrices. While described for completeness, extension of the Justen-Ramlau method to zeropadded BCs is not considered in this work.

(ii) Periodic BCs: The pixels outside of the field of view of the recorded image are assumed to be a periodic extension of the recorded image. Therefore $F_{i, j}=F_{m+i, n+j}$. Periodic boundary conditions are computationally very attractive, since the solution to the deconvolution problem may be computed using fast Fourier transforms. This is due to the fact that the blurring matrix $A$ has a block circulant with circulant block (BCCB) structure. However, methods for image compression and restoration which compute solutions under the assumption of periodic boundary conditions are known to produce oscillatory artifacts in the computed solutions $[17,9]$.

(iii) Reflective BCs, also referred to as reflexive or Neumann BCs: Here it is assumed that all pixels outside of the field of view are mirror reflections of the recorded image about the image boundaries. In other words, $F_{1-i, 1-j}=F_{i, j}$. Reflective boundary conditions do not introduce artificial boundary artifacts, since continuity at each boundary is enforced. This leads to a somewhat complicated structure of the blurring matrix, which takes the form of a sum of four block Toeplitz and block Hankel matrices. Nevertheless, a deconvolution problem involving a symmetric PSF and reflective boundary conditions produce a blurring matrix which may be diagonalized readily through application of the 2D discrete cosine (II) transforms [17]. Further discussion of reflective boundary conditions is provided in Section 4.2.

(iv) Antireflective BCs: Here $F_{1-i, 1-j}=2 F_{1,1}-F_{i+1, j+1}$ and $F_{m+i, n+j}=2 F_{m, n}-$ $F_{m-i, n-j}$. Antireflective boundary conditions enforce continuity of the normal derivative at the boundary and lead to a blurring matrix that can be diagonalized with the aid of the $2 \mathrm{D}$ discrete sine (I) transform and some other (fast) computations. Further discussion of antireflective boundary conditions is given in Section 4.3. A more complete discussion of the algebra of blurring matrices formed from antireflective boundary conditions can be found in [20]. 
Once boundary conditions have been selected, $A$ may be formed, and the solution of (5) can be computed.

\section{The Justen-Ramlau method}

The Justen-Ramlau method is derived under a continuous formulation of the deconvolution problem, and assumes that only the recorded image $g$ and some estimate $\bar{k}$ of the kernel $k$ are available. The method is based upon computing a minimum-norm solution to a closely related problem described in the following definition:

Definition 3. Given a recorded image $g$ along with image and kernel estimators $\bar{f}$ and $\bar{k}$, respectively, the pair $\left(f^{\dagger}(g), k^{\dagger}(g)\right)$ is said to be an $(\bar{f}, \bar{k})$-minimum-norm solution to the problem $f * k=g$ if and only if

$$
\left\|f^{\dagger}-\bar{f}\right\|_{\mathbb{L}^{2}}^{2}+\left\|k^{\dagger}-\bar{k}\right\|_{\mathbb{L}^{2}}^{2}=\min \left\{\|f-\bar{f}\|_{\mathbb{L}^{2}}^{2}+\|k-\bar{k}\|_{\mathbb{L}^{2}}^{2} \mid f, k \in \mathbb{L}^{2}, f * k=g\right\} .
$$

The analysis of the behavior of the minimum-norm solution of Definition 3 is closely related to that of iterative alternating-minimization methods for the functional

$$
F_{\alpha_{1}, \alpha_{2}}(f, k):=\|f * k-g\|_{\mathbb{L}^{2}}^{2}+\alpha_{1}\|f-\bar{f}\|_{\mathbb{L}^{2}}^{2}+\alpha_{2}\|k-\bar{k}\|_{\mathbb{L}^{2}}^{2},
$$

where beginning from estimates $\bar{f}$ and $\bar{k}$ and some initial iterate $f^{0}$, the functional $F_{\alpha_{1}, \alpha_{2}}$ is alternatingly minimized for $k$ and $f$. Here $\alpha_{1}$ and $\alpha_{2}$ are positive constants. This alternating-minimization method is shown to converge to a minimizer pair $(f, k)$ of $F_{\alpha_{1}, \alpha_{2}}$ for $\bar{f}=\bar{k}=0$ and $f^{0}=g$ [6]. Justen and Ramlau [13] showed that when $g$ is produced under the assumption of periodic boundary conditions, the phase of the solution recovered via the alternating-minimization approach is completely determined by the recorded image

$$
\operatorname{sign}(\hat{f}(\omega))=\operatorname{sign}(\hat{g}(\omega))
$$

where $\hat{f}$ and $\hat{g}$ are the Fourier transforms of $f$ and $g$, respectively, and sign denotes the complex sign function. Although a frequency-wise equivalence in phase between the Fourier transform of an exact image and the Fourier transform of a blurry version of itself does not occur in general, it does hold in the case of zero-phase blurring kernels such as Gaussian kernels. In this case, we have

$$
\hat{f}(\omega)=r^{\dagger}(\omega) \hat{g}(\omega)
$$

for some real positive scalar $r^{\dagger}(\omega)$. That is, each Fourier frequency of the blurry image need only be scaled to restore the original image; their phases are equal.

Following Justen and Ramlau [13], we consider the 1D case for notational simplicity with all functions assumed to belong to $\mathbb{L}^{2}(\mathbb{R})$. Using the fact that the Fourier transform is isometric with respect to the $\mathbb{L}^{2}$-norm, the minimum norm solution of Definition 3 satisfies

$$
\|f-\bar{f}\|_{\mathbb{L}^{2}}^{2}+\|k-\bar{k}\|_{\mathbb{L}^{2}}^{2}=\frac{1}{2 \pi} \int_{\mathbb{R}}|\hat{f}(\omega)-\hat{\bar{f}}(\omega)|^{2}+|\hat{k}(\omega)-\hat{\bar{k}}(\omega)|^{2} d \omega .
$$




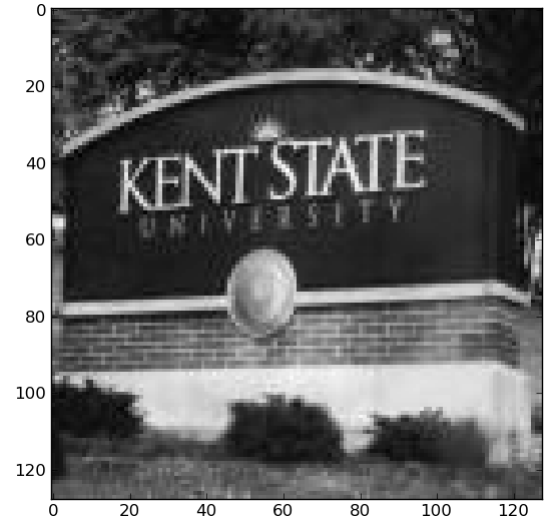

(a) Example image.

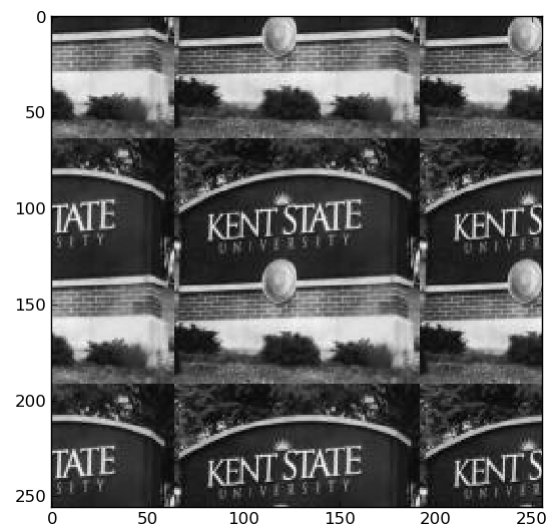

(c) Extension of image assuming periodic BCs.

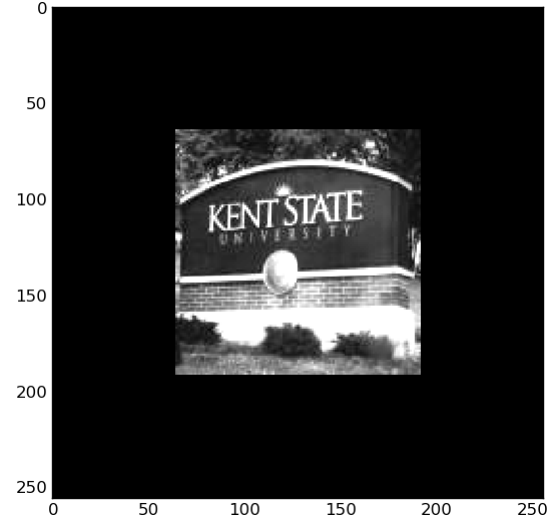

(b) Extension of image assuming zeropadded BCs.

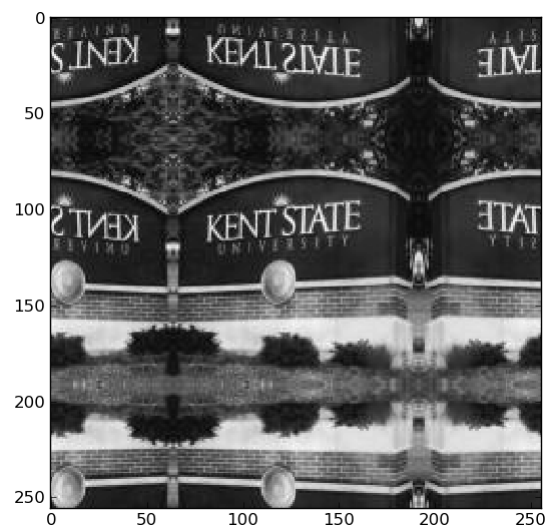

(d) Extension of image assuming reflective BCs.

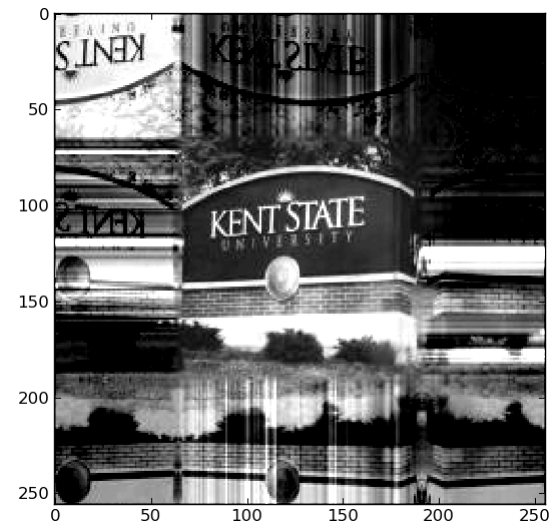

(e) Extension of image assuming antireflective BCs.

Figure 1: An example showing extension of a digital image for a variety of boundary conditions. 
Therefore, it suffices for this functional to be minimized point-wise for each frequency $\omega$

$$
\min _{\hat{f}(\omega), \hat{k}(\omega)}:|\hat{f}(\omega)-\hat{\bar{f}}(\omega)|^{2}+|\hat{k}(\omega)-\hat{\bar{k}}(\omega)|^{2}
$$

with $\hat{f}$ and $\hat{k}$ satisfying the Fourier convolution theorem $\hat{f}(\omega) \hat{k}(\omega)=\hat{g}(\omega)$. In this way, the computation of the minimum-norm solution completely decouples under application of the Fourier transform. Thus, the problem may be restated as

$$
\min _{z, \zeta}:|z-\hat{\bar{f}}(\omega)|^{2}+|\zeta-\hat{\bar{k}}(\omega)|^{2} \text { such that } z \zeta=\hat{g}(\omega),
$$

for each Fourier frequency $\omega$. Since $z \zeta=\hat{g}(\omega)$, we may substitute $\zeta=\frac{\hat{g}(\omega)}{z}$, which gives

$$
\min _{z}:|z-\hat{\bar{f}}(\omega)|^{2}+\left|\frac{\hat{g}(\omega)}{z}-\hat{\bar{k}}(\omega)\right|^{2} .
$$

Justen and Ramlau [13] showed that with the solution estimator $\hat{\bar{f}}(\omega)=\hat{g} \operatorname{sign}^{+}(\hat{\bar{k}}(\omega))$, where

$$
\operatorname{sign}^{+}(\hat{\bar{k}}(\omega))=\left\{\begin{aligned}
1, & \hat{\bar{k}}(\omega) \geqslant 0, \\
-1, & \hat{\bar{k}}(\omega)<0,
\end{aligned}\right.
$$

uniqueness of the solution of (6) is guaranteed for any symmetric, non-negative, and suitably bounded kernel $k$. Substituting this estimator into (7) gives the equivalent problem

$$
\min _{r^{\dagger}(\omega) \geq 0}:\left(r^{\dagger}(\omega)-|\hat{g}(\omega)|\right)^{2}+\left(\frac{|\hat{g}(\omega)|}{r^{\dagger}(\omega)}-|\hat{\bar{k}}(\omega)|\right)^{2}=(r-c)^{2}+\left(\frac{c}{r}-b\right)^{2},
$$

where $r=r^{\dagger}(\omega), c=|\hat{g}(\omega)|>0$ and $b=|\hat{\bar{k}}(\omega)|$ with $0<b<1$. A first-order necessary condition for a scaling factor $r>0$ to minimize this expression is

$$
\frac{d}{d r}\left[(r-c)^{2}+\left(\frac{c}{r}-b\right)^{2}\right]=0
$$

which yields

$$
r^{4}-c r^{3}+b c r-c^{2}=0 .
$$

It is shown in [13] that this polynomial has a unique positive root for $c>0$ and $0 \leq b \leq 1$ defined as above; thus $r^{\dagger}(\omega)$ will be uniquely determined by this method for each $\omega$. Once each $r^{\dagger}(\omega)$ has been found, one may set

$$
\begin{gathered}
\hat{f}^{\dagger}(\omega)=r^{\dagger}(\omega) \hat{g}(\omega) \operatorname{sign}^{+}(\hat{\bar{k}}(\omega)), \\
\hat{k}^{\dagger}(\omega)=\left\{\begin{array}{cc}
\hat{\bar{k}}(\omega), & r=0, \\
\frac{\hat{g}(\omega)}{f^{\dagger}(\omega)}, & r \neq 0,
\end{array}\right.
\end{gathered}
$$

and then compute inverse Fourier transforms of $\hat{f}^{\dagger}$ and $\hat{k}^{\dagger}$ to obtain the minimumnorm solution pair $\left(f^{\dagger}(g), k^{\dagger}(g)\right)$. Finally, Justen and Ramlau [13] considered a generalization based on minimizing functionals of the form

$$
F_{g}(f, k)=\gamma\|f-\bar{f}\|_{\mathbb{L}^{2}}^{2}+\|k-\bar{k}\|_{\mathbb{L}^{2}}^{2}
$$


for some positive scalar $\gamma$. The purpose of this generalization is to rebalance the problem in the case when $\|f\|_{\mathbb{L}^{2}}^{2}$ is much larger than $\|k\|_{\mathbb{L}^{2}}^{2}$. For this, it was shown that the minimum-norm solution is given by

$$
\left(f_{\gamma}^{\dagger}(g), k_{\gamma}^{\dagger}(g)\right)=\left(\frac{1}{\sqrt{\gamma}} f^{\dagger}(\sqrt{\gamma} g), k^{\dagger}(\sqrt{\gamma} g)\right) .
$$

Algorithm 1 below summarizes the Justen-Ramlau method in terms of finitedimensional matrices. One detail worth noting is that in a discrete 2D setting, the discrete convolution theorem requires a circulant shift of the matrix of the pointspread function prior to computation of its Fourier transform [9]. Analysis in [13] shows that for a noise-free problem, no additional regularization is required. In the case where the recorded image is contaminated by additive noise, one may readily apply a spectral filtering method (such as hard thresholding or Tikhonov regularization) to the components of $\hat{g}$. Therefore, inversion and regularization of the blind deconvolution problem exist as separate tasks under this framework. Existence and uniqueness of the minimum-norm solution is only dependent on the choice of periodic boundary conditions through the use of the convolution theorem and Plancherel's formula. We say that a matrix is non-negative if all its entries are non-negative.

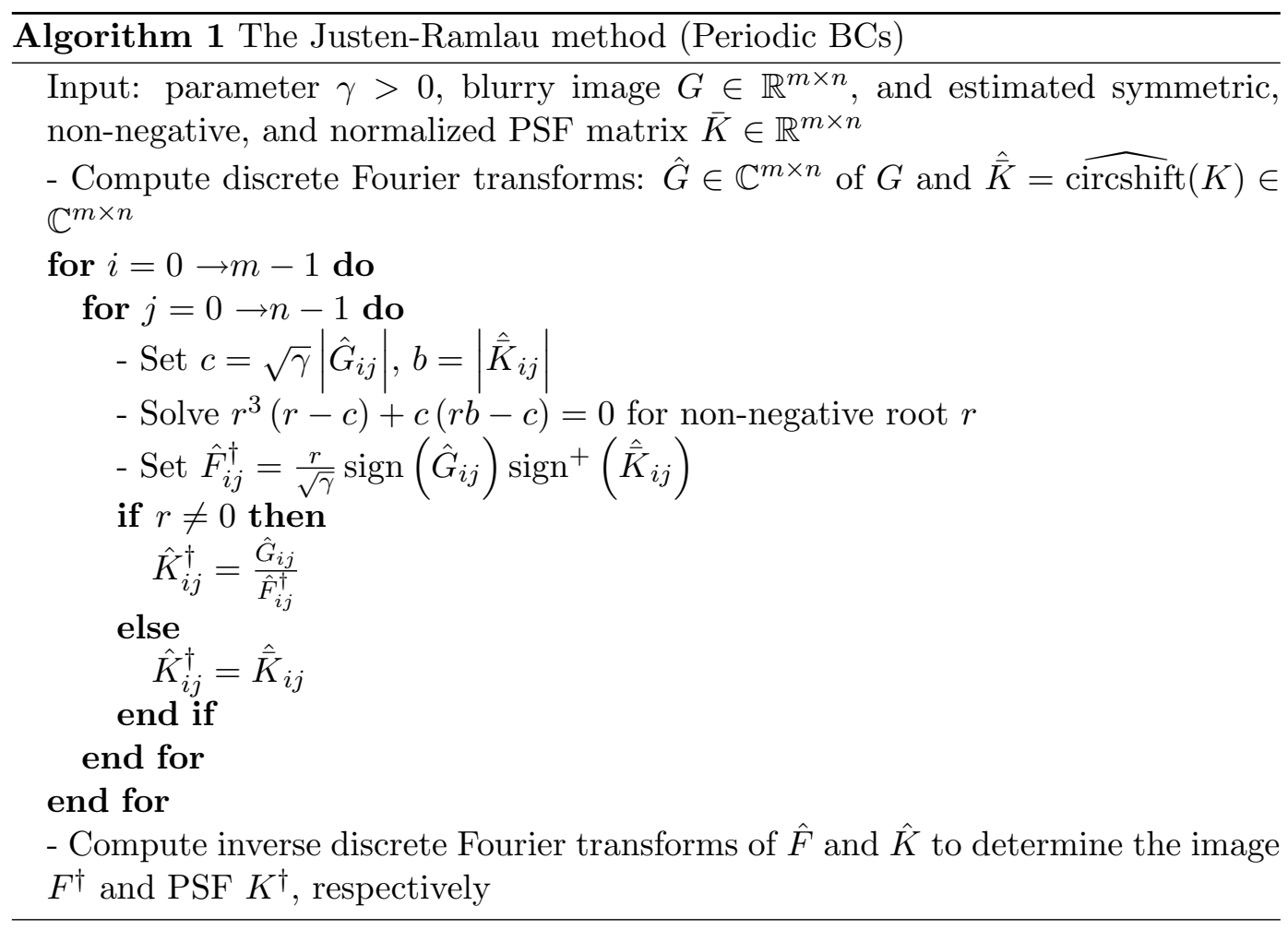

\section{Extension to other BCs}

To extend the Justen-Ramlau method to non-periodic boundary conditions, we have to examine the 2D discrete convolution theorem. The role of the Discrete Fourier 
Transform in the diagonalization of the discrete convolution operator under periodic boundary conditions must be generalized in some way to transformations which diagonalize the convolution operator under other boundary conditions. To this end, we first consider Lemma 4.3.1 in [11].

Lemma 4.1. Let $A \in \mathbb{R}^{m \times m}, B \in \mathbb{R}^{n \times n}$, and $x \in \mathbb{R}^{m n}$. Then $(A \otimes B) x=$ vec $\left(B \operatorname{mat}(x) A^{T}\right)$, where $A \otimes B$ represents the Kronecker product of $A$ and $B$.

This lemma shows that forward multiplication of a Kronecker product with a vectorized matrix can be interpreted as a vectorization of a similar transformation applied to the rows and columns of the matrix directly. As an example, this duality can be applied to the 2D Discrete Fourier Transform. The 2D Discrete Fourier Transformation of a matrix can either be described as multiplication of a vectorization of this matrix with the Kronecker product of two 1D Discrete Fourier Transform matrices, or simply as a 1D Discrete Fourier Transform applied to each row of the matrix, followed by the transformation of each column. Analogous forward transformations arise when considering the diagonalization of the matrices of certain blurring operators. When this happens, it can be shown that a decoupling relationship exists between the pixels of a transformed blurred image and the corresponding pixels of the transformed exact image. A proof of this decoupling relationship is given next.

Theorem 4.2. Consider equation (5), where $b=\operatorname{vec}(G)$ and $x=\operatorname{vec}(F)$ for the blurred image matrix $G$ and the exact image matrix $F$. Assume the boundary conditions to be such that the matrix $A \in \mathbb{R}^{m n \times m n}$ is normal. Then $A A^{*}=A^{*} A$ and

$$
A=U^{*} \Lambda U
$$

for some unitary matrix $U \in \mathbb{C}^{m n \times m n}$ and diagonal eigenvalue matrix $\Lambda \in \mathbb{C}^{m n \times m n}$. Furthermore, assume that $U$ may be factored as a Kronecker product

$$
U=V_{m \times m} \otimes V_{n \times n},
$$

where $V_{m \times m} \in \mathbb{C}^{m \times m}$ and $V_{n \times n} \in \mathbb{C}^{n \times n}$ are unitary matrices. Let $a_{1} \in \mathbb{R}^{m n}$ represent the first column of $A, e_{1}$ represent the first vector of the canonical basis of $\mathbb{R}^{m n}$, and $M \in \mathbb{R}^{m \times n}$ be a matrix containing the eigenvalues of $A$,

$$
M=\operatorname{mat}\left(\Lambda \sum_{i=1}^{m n} e_{i}\right)
$$

i.e., the matrix $M$ has the entries

$$
M_{i, j}=\Lambda_{(j-1) n+i,(j-1) n+i} .
$$

Then the components of $\hat{F}, \hat{G}$, and $M$ obey the decoupling relationship

$$
\hat{G}_{i, j}=\hat{F}_{i, j} \frac{\left.\widehat{\operatorname{mat}\left(a_{1}\right.}\right)_{i, j}}{\widehat{\operatorname{mat}\left(e_{1}\right)_{i, j}}}=\hat{F}_{i, j} M_{i, j}, \quad 1 \leq i \leq m, \quad 1 \leq j \leq n,
$$

where $\hat{H}$ denotes the transformation $\hat{H}=\operatorname{mat}(\operatorname{Uvec}(H))$ of a matrix $H \in \mathbb{R}^{m \times n}$. 
Proof. We have

$$
U A=\Lambda U \Rightarrow U a_{1}=\Lambda U e_{1},
$$

where $a_{1}$ is the first column of $A$ and $e_{1}=[1,0, \ldots, 0]^{T}$ is the first vector of the canonical basis of $\mathbb{R}^{m n}$. Solving for each eigenvalue $\lambda_{i}$ gives

$$
\lambda_{i}=\frac{\left[U a_{1}\right]_{i}}{\left[U e_{1}\right]_{i}}
$$

where $[z]_{i}$ denotes the $i^{\text {th }}$ component of the vector $z$. For equation (5), we have $x, b \in \mathbb{R}^{m n}$ as vector representations of the matrices $F, G \in \mathbb{R}^{m \times n}$ created by stacking their respective columns (as discussed in Section 2). These relationships can be written as

$$
\begin{aligned}
b=\operatorname{vec}(G) & \Leftrightarrow \quad G=\operatorname{mat}(b), \\
x=\operatorname{vec}(F) & \Leftrightarrow \quad F=\operatorname{mat}(x) .
\end{aligned}
$$

Therefore, the $m n$ eigenvalues may be collected in the matrix $M$ with entries

$$
M_{i, j}=\frac{\operatorname{mat}\left(U a_{1}\right)_{i, j}}{\operatorname{mat}\left(U e_{1}\right)_{i, j}} .
$$

By (5) and (8), we have $U b=\Lambda U x$, and application of Lemma 4.1, using (9), yields

$$
\begin{aligned}
& U a_{1}=\left(V_{m \times m} \otimes V_{n \times n}\right) a_{1}=\operatorname{vec}\left(V_{n \times n} \operatorname{mat}\left(a_{1}\right) V_{m \times m}^{T}\right) \\
& U e_{1}=\left(V_{m \times m} \otimes V_{n \times n}\right) e_{1}=\operatorname{vec}\left(V_{n \times n} \operatorname{mat}\left(e_{1}\right) V_{m \times m}^{T}\right) .
\end{aligned}
$$

It follows that we can write the forward discrete transformations $U a_{1}, U e_{1}, U b$, and $U x$ as forward $2 \mathrm{D}$ orthogonal transformations applied directly to their matrix representations:

$$
\begin{aligned}
\operatorname{mat}\left(U a_{1}\right) & =\operatorname{mat}\left(\hat{a}_{1}\right)=\widehat{\operatorname{mat}\left(a_{1}\right)}, \\
\operatorname{mat}\left(U e_{1}\right) & =\operatorname{mat}\left(\hat{e}_{1}\right)=\widehat{\operatorname{mat}\left(e_{1}\right)}, \\
\operatorname{mat}(U b) & =\operatorname{mat}(\hat{b})=\hat{\operatorname{mat}(b)}=\hat{G}, \\
\operatorname{mat}(U x) & =\operatorname{mat}(\hat{x})=\hat{\operatorname{mat}(x)}=\hat{F} .
\end{aligned}
$$

Since $U$ is a unitary matrix, this transformation is isometric with respect to the 2norm. This provides the component-wise decoupling of the deconvolution problem (10).

For our purposes, equation (10) may be interpreted as a generalization of the Fourier convolution theorem for the applied boundary conditions. It must be noted, however, that not all boundary conditions used in digital image deblurring problems correspond to a normal blurring matrix. 


\subsection{Generalizing the Justen-Ramlau method}

The following analysis of our generalization of the Justen-Ramlau method is restricted to finite-dimensional linear algebra for simplicity, i.e., we let $F, K, G \in \mathbb{R}^{m \times n}$ and $A \in \mathbb{R}^{m n \times m n}$. Extension to infinite-dimensional Hilbert spaces is straightforward.

Assume that the boundary conditions and the PSF matrix $K$ are such that the blurring matrix admits a spectral factorization (8) with an eigenvector matrix that satisfies (9). Also let the kernel matrix $K$ be symmetric, non-negative, and normalized as described below. Similarly as in the previous section, let $U$ define a forward discrete $2 \mathrm{D}$ orthogonal transform $\hat{X}$ for any matrix $X \in \mathbb{R}^{m \times n}$. Then the decoupling relationship

$$
\hat{G}_{i, j}=\hat{F}_{i, j} M_{i, j}
$$

holds as discussed in the previous section. Let an estimate of the eigenspectrum $\bar{M}$ be given by

$$
\bar{M}_{i, j}=\frac{\left.\widehat{\operatorname{mat}\left(\bar{a}_{1}\right.}\right)_{i, j}}{\widehat{\operatorname{mat}\left(e_{1}\right)_{i, j}}},
$$

where $\bar{a}_{1}$ is the first column of the blurring matrix $\bar{A}$, determined from the chosen kernel estimate $\bar{K}$ using the selected BCs. Note that it may not be necessary to construct $\bar{A}$ in order to have access to the components of $\bar{a}_{1}$, because

$$
\bar{a}_{1}=\bar{A} e_{1}=\bar{A} \operatorname{vec}\left(\left[\begin{array}{cccc}
1 & 0 & \cdots & 0 \\
0 & 0 & & \vdots \\
\vdots & & \ddots & \vdots \\
0 & \ldots & \cdots & 0
\end{array}\right]\right)
$$

i.e., the first column of $\bar{A}$ is the vectorization of a unit impulse response of $\bar{A}$, centered at the first row and first column with values wrapped around the matrix according to the specified boundary conditions. By definition, the unit impulse response of $\bar{A}$ is exactly the PSF matrix $\bar{K}$. Thus, if the boundary conditions are such that all pixels of $\bar{K}$ are available after wrapping (such as in the case of periodic boundary conditions), then all of the values of $\bar{K}$ are explicitly available in $\bar{a}_{1}$ and vice-versa. If the boundary conditions are such that only a portion of $\bar{K}$ remains after wrapping (such as in the case of reflective and antireflective BCs), then the values of $\bar{K}$ are only known completely in $\bar{a}_{1}$ if $\bar{K}$ obeys some symmetry conditions. Thus, certain combinations of boundary conditions and symmetric PSF matrices allow for $\bar{a}_{1}$ to be easily constructable from the components of $\bar{K}$. This is examined further in Sections 4.2 and 4.3.

Consider the matrix functional

$$
\mathcal{F}(F, M)=\|F-\bar{F}\|_{2}^{2}+\|M-\bar{M}\|_{2}^{2},
$$

where $\bar{F}$ is an a priori estimate for the exact image $F$ and $\bar{M}$ is an estimate of the matrix $M$ of eigenvalues of the blurring operator. From this, we may define a minimum-norm solution for the problem of blind deconvolution of $G$ with eigenvalue matrix estimate $\bar{M}$ under the assumptions stated above to be the matrix pair 
$\left(F^{\dagger}, M^{\dagger}\right)$ such that

$$
\mathcal{F}\left(F^{\dagger}, M^{\dagger}\right)=\min _{F, M \in \mathbb{R}^{m \times n}}\left\{\mathcal{F}(F, M) \mid \hat{G}_{i, k}=\hat{F}_{i, k} M_{i, k}, 1 \leqslant i \leqslant m, 1 \leqslant j \leqslant n\right\} .
$$

We may now proceed to directly solve for a minimum-norm solution as before. However, note that in this case, we are not recovering an estimate of the exact point spread function $K^{\dagger}$ but instead an estimated matrix of eigenvalues of $A$, denoted by $M^{\dagger}$. The following theorem discusses the solution of this minimization problem. The condition on the eigenvalues of $A$ will be commented on below.

Theorem 4.3. Let $G \in \mathbb{R}^{m \times n}$ be a recorded blurry digital image with $\bar{K} \in \mathbb{R}^{m \times n}$ being a symmetric, non-negative, bounded, estimate of the associated blurring kernel. Assume that the applied boundary conditions and blurring kernel are such that the blurring operator $A \in \mathbb{R}^{m n \times m n}$ is a normal matrix with spectral factorization $A=$ $U^{*} \Lambda U$, where $U$ admits a Kronecker product factorization $U=V_{m \times m} \otimes V_{n \times n}$ and the eigenvalues are bounded by one in magnitude. Let the solution estimator be

$$
\hat{F}_{i, k}=r^{\dagger}(\omega)_{i, j} \hat{G}_{i, k} \operatorname{sign}^{+}\left(\bar{M}_{i, k}\right) .
$$

Then there exists a unique minimum-norm solution $\left(F^{\dagger}, M^{\dagger}\right)$ of (11).

Proof. Just as in the Justen-Ramlau method, a complex sign invariance is assumed to hold between the components of $\hat{F}$ and $\hat{G}$, so that we may proceed by searching for $\hat{F}$ such that (12) holds for each component $\hat{F}_{i, j}$. Isometry of the forward transform defined by $U$ with respect to the 2-norm implies that for each $1 \leq i \leq m, 1 \leq j \leq n$, a minimum-norm solution must minimize

$$
\min _{z, \zeta}:\left|z-\hat{\bar{F}}_{i, j}\right|^{2}+\left|\zeta-\bar{M}_{i, j}\right|^{2} \text { such that } z \zeta=\hat{G}_{i, k}
$$

or, equivalently,

$$
\min _{z}:\left|z-\hat{\bar{F}}_{i, j}\right|^{2}+\left|\frac{\hat{G}_{i, k}}{z}-\bar{M}_{i, j}\right|^{2} .
$$

By assumption, for each $i, k$, we are searching for $z$ such that

$$
z=r^{\dagger}(\omega)_{i, j} \hat{G}_{i, j} \operatorname{sign}^{+}\left(\bar{M}_{i, j}\right)
$$

with initial image estimate $\hat{\bar{F}}_{i, k}=\hat{G}_{i, j} \operatorname{sign}^{+}\left(\bar{M}_{i, j}\right)$. Hence, it suffices to solve

$$
\min _{r \geq 0}:\left(r-\left|\hat{G}_{i, k}\right|\right)^{2}+\left(\frac{\left|\hat{G}_{i, k}\right|}{r}-\left|\bar{M}_{i, j}\right|\right)^{2} .
$$

By assumption, $\left|\bar{M}_{i, j}\right| \leqslant 1$. Therefore, the search for a minimum-norm solution under general boundary conditions simplifies to the search for a positive root of a quartic polynomial of the same form as in the Justen-Ramlau method with periodic boundary conditions. Since this polynomial has been shown to possess exactly one positive root for each possible $\left|\hat{G}_{i, k}\right|$ and $\left|\bar{M}_{i, k}\right|$, each component of $F^{\dagger}$ and $M^{\dagger}$ is completely determined; thus, a unique minimum-norm solution exists. 
The following corollary provides sufficient conditions for the eigenvalues of the matrix $A$ in Theorem 4.3 to be of magnitude at most one. Other sufficient conditions also can be formulated. We comment on the conditions of the corollary in our discussions of the boundary conditions below.

Corollary 4.1. Let $G \in \mathbb{R}^{m \times n}$ be a recorded blurry digital image and let $\bar{K} \in \mathbb{R}^{m \times n}$ be a symmetric estimate of the associated blurring kernel such that

$$
\sum_{i=1}^{m} \sum_{j=1}^{n}\left|\bar{K}_{i, j}\right| \leqslant 1 .
$$

Assume that the blurring operator $A \in \mathbb{R}^{m n \times m n}$ that determines $G$ satisfies

$$
\|A\|_{\infty} \leqslant \sum_{i=1}^{m} \sum_{j=1}^{n}\left|\bar{K}_{i, j}\right|,
$$

where $\|\cdot\|_{\infty}$ denotes the matrix norm induced by the uniform vector norm in $\mathbb{R}^{m n}$. Then the eigenvalues of $A$ are bounded by one in magnitude.

Proof. The result follows from the fact that the spectral radius of $A$ is bounded by $\|A\|_{\infty}$.

The exact instances in which the assumed complex sign invariance occurs in practice is a subject worthy of further study. A sufficient condition would be for the exact blurring matrix $A$ to be positive definite. This would imply that $M_{i, j}>0$ for each $1 \leq i \leq m$ and $1 \leq j \leq n$, which would give a component-wise sign invariance

$$
\operatorname{sign}\left(\hat{G}_{i, k}\right)=\operatorname{sign}\left(\hat{F}_{i, j} \bar{M}_{i, j}\right)=\operatorname{sign}\left(\hat{F}_{i, k}\right),
$$

as required. However, this condition is not assumed here, only that the relationship (12) holds.

We have observed that when the blurring matrix is far from normal and has complex eigenvalues, our restoration method may yield poor images. This is the situation when the available image is contaminated by motion blur.

The condition (14) is satisfied when each row of $A$ represents some combination of the components of $\bar{K}$ (without duplicates). This is clearly satisfied under periodic boundary conditions for any $\bar{K}$, due to the block circulant structure which emerges for the blurring matrix $A$.

It is straightforward to extend Theorem 4.3 to allow for a trade-off parameter $\gamma$ for the case when $\|F\|_{2} \gg\|M\|_{2}$. This extension is used in Algorithm 2, which describes our method.

\subsection{Reflective boundary conditions}

Consider a deconvolution problem with reflective boundary conditions. Assume that the PSF matrix obeys the symmetry conditions

$$
K_{i, j}=K_{-i, j}=K_{i,-j}=K_{-i,-j} .
$$




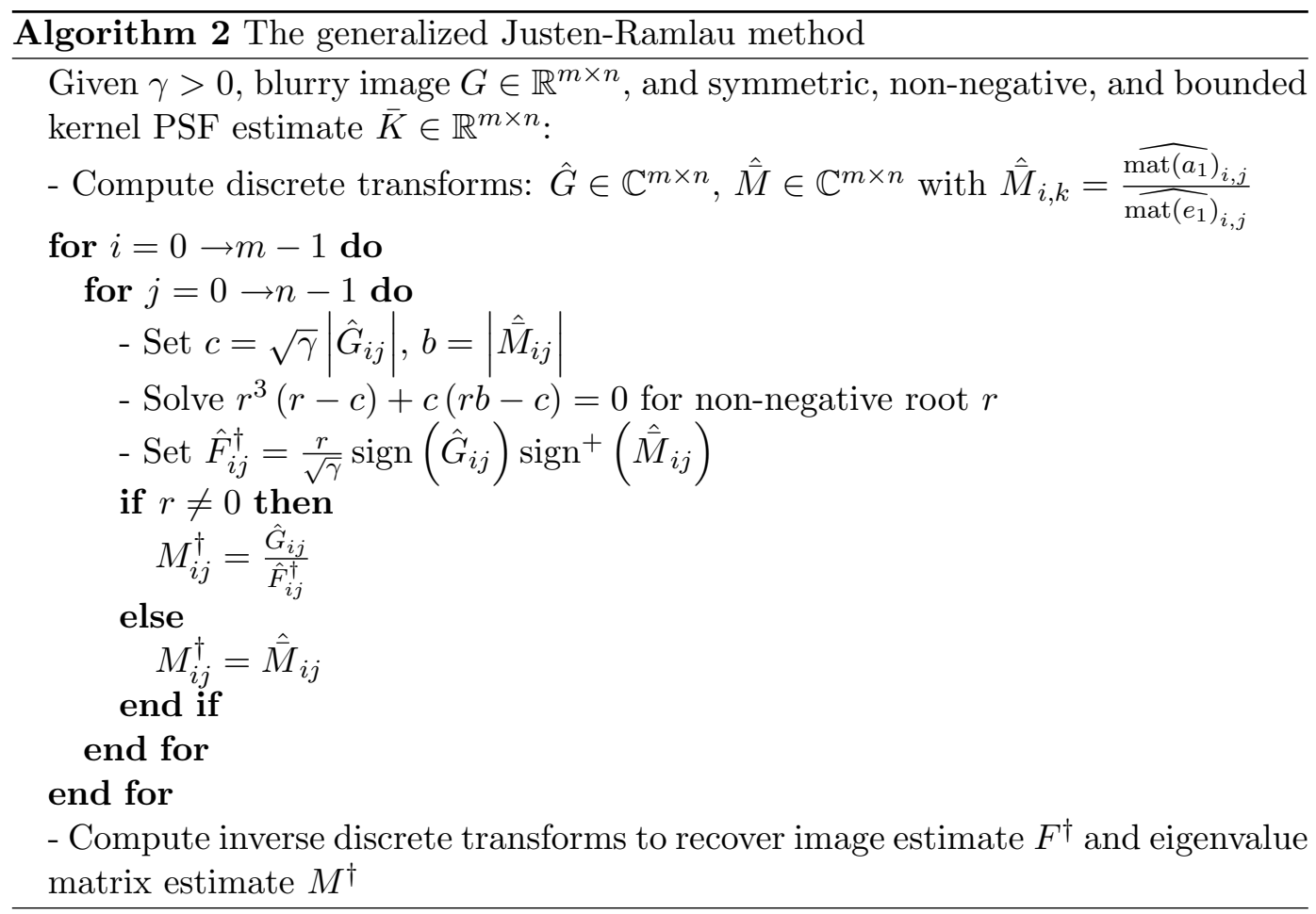

Then the matrix $A$ is normal. It is shown in [17] that $A$ is the sum of a blockToeplitz-Toeplitz-block matrix, a block-Toeplitz-Hankel-block matrix, a block-HankelToeplitz-block matrix, and a block-Hankel-Hankel-block matrix, and that matrices of this type admit the spectral factorization

$$
A=C^{T} \Lambda C,
$$

where $C \in \mathbb{R}^{m n \times m n}$ is the matrix of the $2 \mathrm{D}$ discrete cosine transform. The matrix $C$ can be factored as

$$
C=\mathcal{C}_{m} \otimes \mathcal{C}_{n},
$$

where $\mathcal{C}_{m} \in \mathbb{R}^{m \times m}$ and $\mathcal{C}_{n} \in \mathbb{R}^{n \times n}$ are $1 \mathrm{D}$ discrete cosine transform matrices. The components of $\mathcal{C}_{n}$ are given by

$$
\left[\mathcal{C}_{n}\right]_{i, j}=\left\{\begin{array}{cl}
\sqrt{\frac{1}{n}}, & i=0, \\
\sqrt{\frac{2}{n}} \cos \left(\frac{(i-1)(2 j-1) \pi}{2 n}\right), & i \neq 0 .
\end{array}\right.
$$

The assumed symmetry condition implies that each row of $A$ represents a combination of the elements of $K$. Therefore, the bound (14) holds. It follows that deblurring problems under reflective boundary conditions with PSF matrices obeying equation (15) fall within the framework of the extended Justen-Ramlau method.

Let

$$
\hat{B}=\operatorname{DCT} 2 \mathrm{D}(B)
$$

represent the discrete cosine transform of a matrix $B$. After selection of a PSF estimate $\bar{K}$, one may begin construction of the estimated eigenvalue matrix $\bar{M}$ by 
first computing mat $\left(e_{1}\right)_{i, j}$ directly. The components of $A$ can be computed from the components of $\bar{K}$, without requiring the explicit construction of $A$; see, e.g., [9]. This is done by shifting the values of the pixels in the PSF matrix so that the center pixel is the value of the first entry of the matrix (first row and first column). Rather than wrapping the pixel values circulantly around the matrix, the pixel values are wrapped according to reflexivity of the boundary conditions and bounded to satisfy (13). This gives the matrix mat $\left(a_{1}\right)$. The matrix mat $\left(a_{1}\right)$ can be computed as

$$
\operatorname{mat}\left(a_{1}\right)=Z_{1} \bar{K} Z_{1}^{T}+Z_{1} \bar{K} Z_{2}^{T}+Z_{2} \bar{K} Z_{1}^{T}+Z_{2} \bar{K} Z_{2}^{T}
$$

where $Z_{1}$ and $Z_{2}$ are shift matrices. If the estimated PSF $\bar{K}$ has its center at pixel $(i, i)$, then $Z_{1}$ is the shift matrix with components given by

$$
\left(Z_{1}\right)_{i, j}= \begin{cases}1, & j=i+1 \\ 0, & \text { otherwise }\end{cases}
$$

and $Z_{2}$ has the components

$$
\left(Z_{2}\right)_{i, j}= \begin{cases}1, & j=i+2 \\ 0, & \text { otherwise }\end{cases}
$$

see [9] for further details. Once the matrix of estimated eigenvalues $\bar{M}$ is available, the computation of $\left(F^{\dagger}, M^{\dagger}\right)$ proceeds just as in Algorithm 2.

\subsection{Antireflective boundary conditions}

Consider an image deblurring problem in which antireflective boundary conditions have been assumed. Just as in the case of reflective boundary conditions, assume that the PSF matrix $K$ obeys the symmetry condition (15). Then the blurring matrix $A$ will generally fail to be normal, but will have the diagonalizable structure

$$
A=\left[\begin{array}{ccccc}
z_{1}+a_{0} & 0 & \cdots & 0 & 0 \\
z_{2}+a_{1} & & & & 0 \\
\vdots & & & & a_{m} \\
z_{m}+a_{m-1} & & \tilde{A} & & z_{m}+a_{m-1} \\
a_{m} & & & & \vdots \\
0 & & & & z_{2}+a_{1} \\
0 & 0 & \cdots & 0 & z_{1}+a_{0}
\end{array}\right]
$$

where each $a_{j}, z_{j}$ are block matrices and $\tilde{A}$ is a highly structured sum of a Toeplitz matrix, a Hankel matrix, and a rank-two correction matrix. This matrix has a block structure that is diagonalized by the discrete sine (I) transform (DST), which may be implemented essentially through use of the fast Fourier transform; see SerraCapizzano [20]. A linear transformation may be defined to compute the eigenvalues of $A$ using the components of $G$ and $K$ without explicit construction of $A$, defined mostly through use of the DST; see [20, 1] for details. However, it does not follow from the definition of the boundary conditions that the bound (14) holds for symmetric, non-negative, and suitably bounded kernels. It is also important to note that this transformation fails to be unitary, since the first and last columns of the matrix 

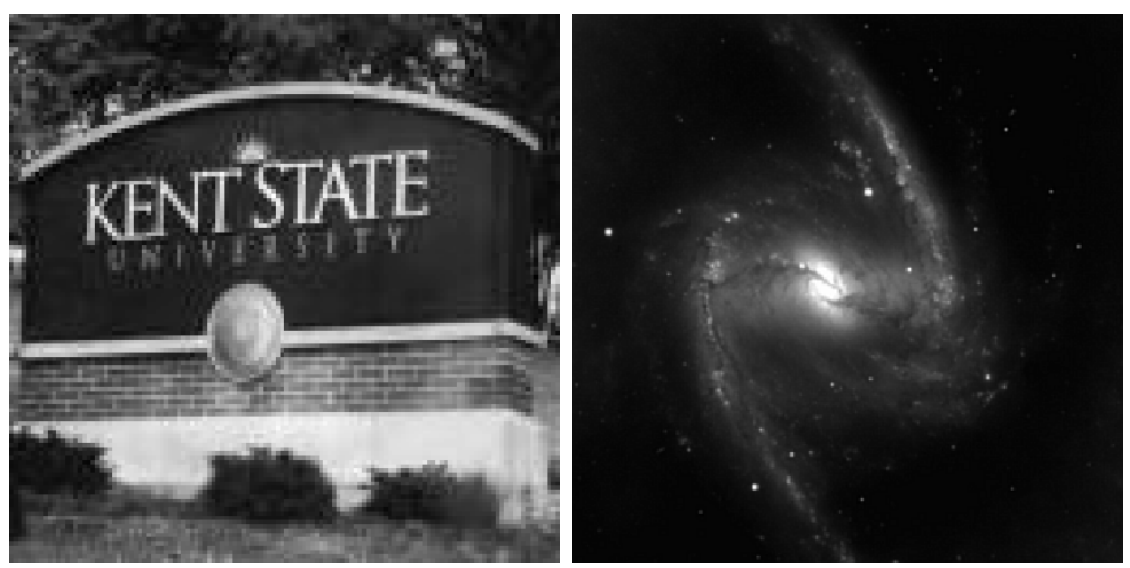

Figure 2: The original unblurred Kent and Galaxy images.

that defines this transformation are not necessarily orthogonal. However, pairwise orthogonality holds for all other columns of this matrix. Thus, if it is assumed that

$$
\underset{F, M}{\arg \min }\left\{\|F-\bar{F}\|_{2}^{2}+\|M-\bar{M}\|_{2}^{2}\right\}=\underset{\hat{F}, M}{\arg \min }\left\{\|\hat{F}-\hat{\bar{F}}\|_{2}^{2}+\|M-\bar{M}\|_{2}^{2}\right\}
$$

and that the spectrum of $A$ is inside the unit circle, then one may still consider searching for an $(\bar{F}, \bar{M})$ minimum-norm solution $\left(F^{\dagger}, M^{\dagger}\right)$ to $\mathcal{F}(F, M)$ through component-wise minimization under application of the forward $2 \mathrm{D}$ antireflective transformation. With these assumptions, extension of the Justen-Ramlau method to antireflective BCs follows.

\section{$5 \quad$ Numerical examples}

In this section, numerical examples of the extended Justen-Ramlau method are computed. All examples have been computed using code implemented in the Python ${ }^{1}$ programming language, making use of the $\mathrm{Numpy}^{2}, \mathrm{Scipy}^{3}$, and Matplotlib ${ }^{4}$ packages.

\section{$5.1 \quad$ Test images}

The two test images used in the numerical examples are the $128 \times 128$ pixel Kent image and the $304 \times 304$ pixel Galaxy image. The full exact images are each shown in Figure 2. When the exact image $F$ is available, the quality of a restored image $F^{\dagger}$ can be measured by the Peak Signal-To-Noise Ratio (PSNR) defined for 8-bit gray-scale images as

$$
\operatorname{PSNR}\left(F, F^{\dagger}\right)=20 \log _{10}\left(\frac{255}{\operatorname{RMSE}\left(F, F^{\dagger}\right)}\right)
$$

\footnotetext{
${ }^{1}$ www.python.org

${ }^{2}$ www.numpy.scipy.org

${ }^{3}$ www.scipy.org

${ }^{4}$ www.matplotlib.sourceforge.net
} 

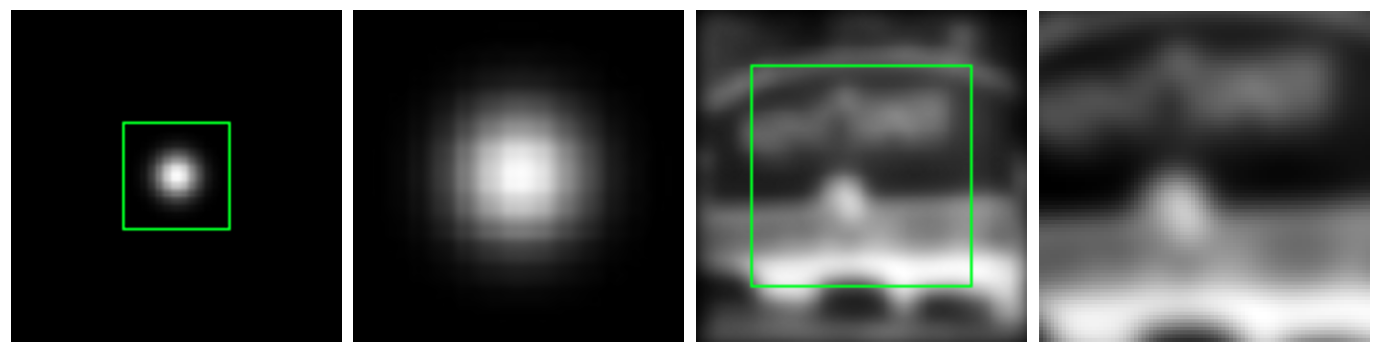

Figure 3: Illustration of the Independence of the central portions of a blurred image when the nonzero part of the PSF matrix is confined to a small central region.

where RMSE $\left(F, F^{\dagger}\right)$ is the Root Mean Squared Error function. It is computed as

$$
\operatorname{RMSE}\left(F, F^{\dagger}\right)=\sqrt{\frac{1}{m n} \sum_{i, j}\left(F_{i, j}-F_{i, j}^{\dagger}\right)^{2}},
$$

where $F, F^{\dagger}$ have dimensions $m \times n$, as above. We used the PSNR to measure the quality of the restored images.

\subsection{Creating blurred images}

To illustrate how realistic test data can be created in image deblurring problems, consider a problem in which the non-zero components of the PSF matrix $K$ are concentrated in a small portion near the center of the matrix. Many PSF matrices, such as those corresponding to mild Gaussian blurring, essentially have this property. Next, consider the largest $p \times q$ submatrix centered at the center of the PSF such that the component of smallest magnitude is of magnitude larger than some chosen threshold. In such a case, the values of pixels within the central region of the resulting blurred image contain negligible contribution from pixels outside of the field of view of the image inferred by the boundary conditions. This region is the $(m-p) \times(n-q)$ submatrix at the center of the matrix $G=K * F$ representing the blurry image. Thus, the components of this submatrix of the blurred image will change only negligibly if blurred using different boundary conditions. Perhaps more importantly, the possible solutions of a deblurring problem posed using only this central region as a test image will be unbiased to the particular BCs used to construct the image. In this way, one can create blurred images which are realistic approximations of blurred images taken from some infinite scene [9]. Figure 3 illustrates this principle. Shown in this figure are a full PSF, a central submatrix extracted from the full PSF, a full-sized image blurred using the full PSF, and the central portion of the blurred image whose values are nearly $\mathrm{BC}$ independent. The (green) rectangles in two of the above images show where the image directly to the right is extracted. All numerical examples which follow are computed used blurred images synthetically generated in this manner.

\subsection{Test PSFs}

As a blind deconvolution method, it is worth testing the quality of the recovered minimum-norm solution when the estimated PSF is of a type different from the 
exact PSF used in the construction of the blurred image. To this end, three separate types of PSFs are used in the numerical examples which follow. Each PSF type is parametrized by some specified positive scalar $\sigma$. A standard Gaussian blur PSF can be defined by constructing the matrix

$$
\tilde{K}_{i, j}=\exp \left\{-\frac{1}{2 \sigma^{2}}\left[\left(i-\frac{m}{2}\right)^{2}+\left(j-\frac{n}{2}\right)^{2}\right]\right\} .
$$

In a similar way, a PSF constructed as a $2 \mathrm{D}$ extension of the density function of the Laplacian probability distribution can be constructed using the matrix whose components are given by

$$
\tilde{K}_{i, j}=\frac{1}{2 \sigma} \exp \left\{-\frac{1}{\sigma}\left[\left|i-\frac{m}{2}\right|+\left|j-\frac{n}{2}\right|\right]\right\} .
$$

Finally, we determine a PSF using the Cauchy probability distribution. In this case, we construct the matrix

$$
\tilde{K}_{i, j}=\left(\pi \sigma\left[1+\frac{1}{\sigma^{2}}\left(i-\frac{m}{2}\right)^{2}+\frac{1}{\sigma^{2}}\left(j-\frac{n}{2}\right)^{2}\right]\right)^{-1} .
$$

In each case, the resulting PSF is normalized as

$$
K_{i, k}=\frac{\tilde{K}_{i, j}}{\sum_{i, j} \tilde{K}_{i, j}}
$$

Figure 4 shows $80 \times 80$ pixel examples of each PSF type, as well as a plot comparing cross sections of each. These cross sections are given by a column near the center of each PSF. Note the difference in peakedness exhibited by the Gaussian and Cauchy PSFs when compared with the Laplacian PSF. Note also the thickness of the tails of the Cauchy PSF and Laplacian PSFs versus the Gaussian PSF.

\subsection{Example of non-blind deconvolution}

The sensitivity of non-blind deconvolution to variation in the PSF is demonstrated next, to motivate the topic of blind deconvolution. Consider a case where an image has been blurred with a Laplacian PSF (with no additive noise), but is to be restored using a Gaussian PSF (via Tikhonov regularization). That is, the restored image was computed by solving

$$
\hat{x}=\underset{x}{\arg \min }\left\{\|A x-b\|_{2}^{2}+\alpha\|x\|_{2}^{2}\right\},
$$

with $A, x$ and $b$ defined as in (5). The regularization parameter $\alpha>0$ was chosen to provide the most visually accurate solution. The result is shown in Figure 5. The sensitivity of deconvolution to changes in the structure of the PSF is apparent in Figure 5; the optimal restoration has severe artifacts and is unfocused. 


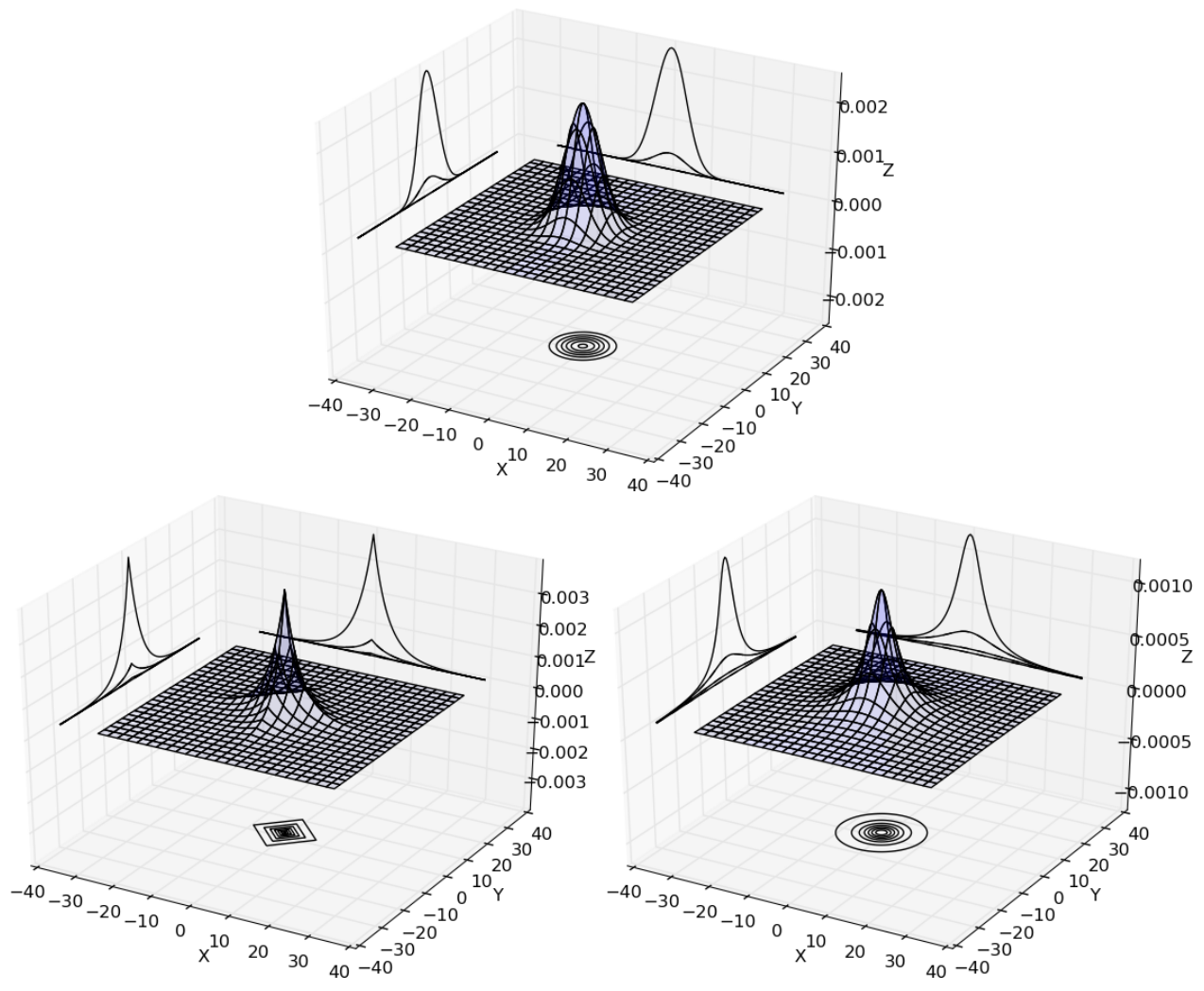

Figure 4: Examples of Gaussian (top), Laplacian (left), and Cauchy (right) PSFs as well as their central cross-sections and contours.
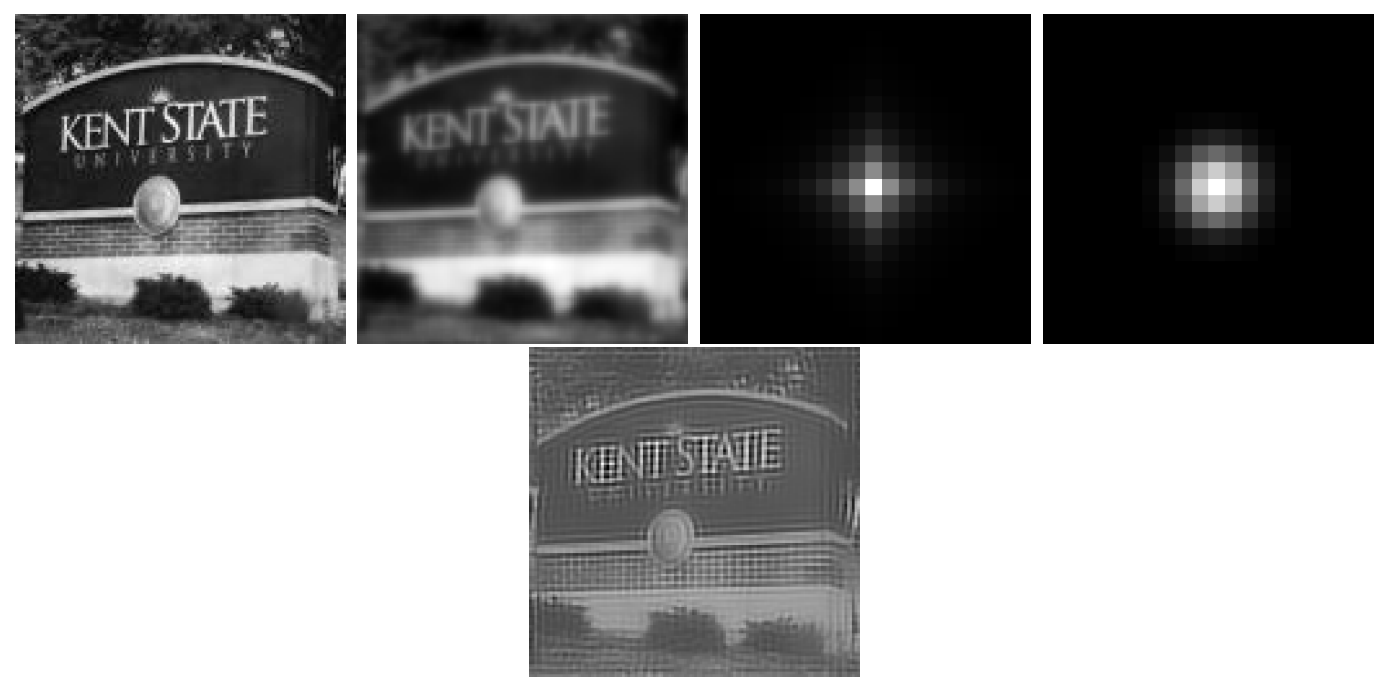

Figure 5: An example of a restoration computed using an incorrect PSF. The top row (from left to right) shows the exact image, the blurred images, the exact PSF, and the assumed PSF. The image in the second row shows the optimal Tikhonov regularized restoration, using the assumed PSF. 

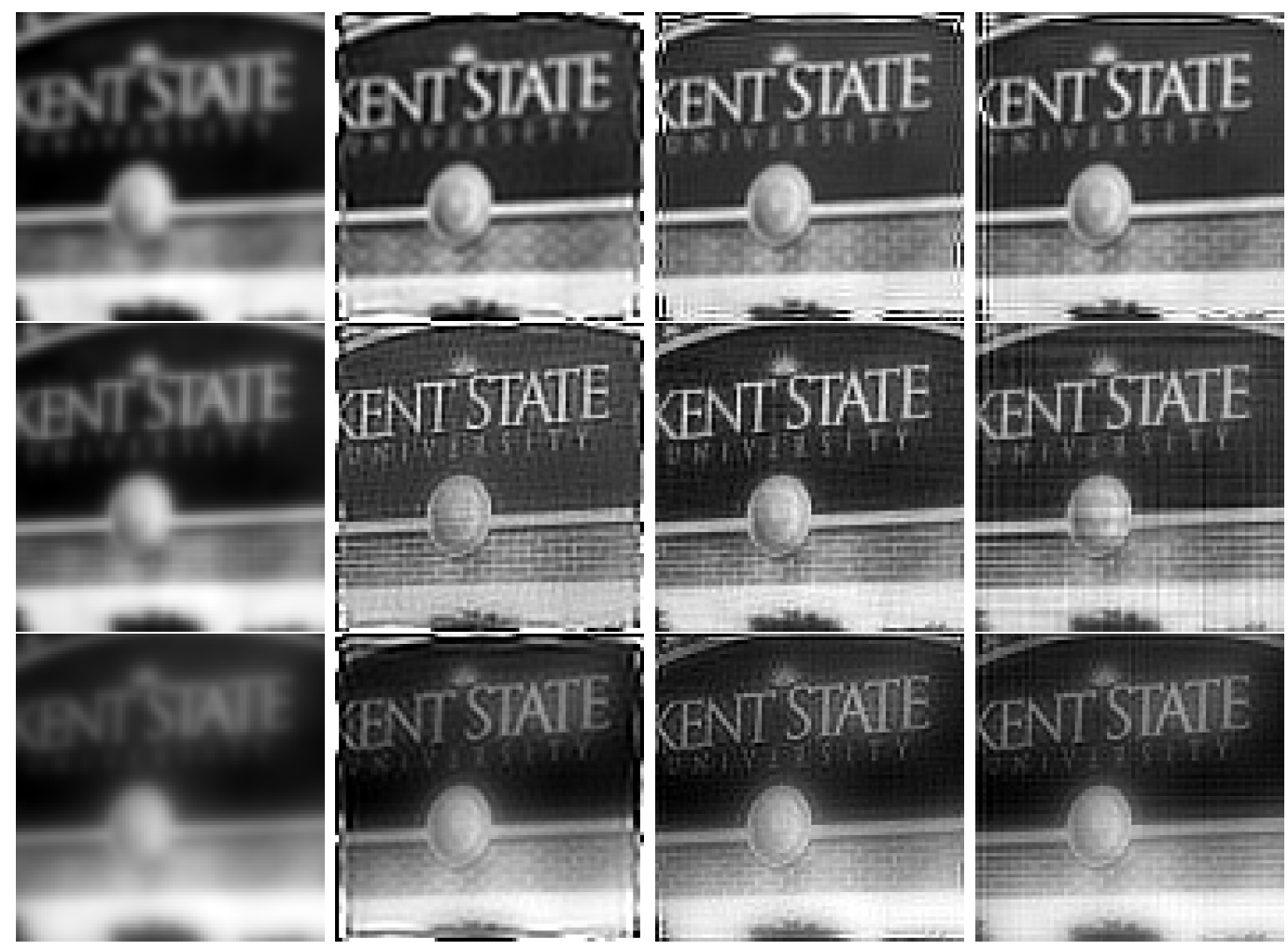

Figure 6: Kent image subjected to mild blurring, and the corresponding restorations. Rows 1 through 3 represent blurred images constructed using Gaussian, Laplacian, and Cauchy PSFs, respectively. The first column of each row represents the blurred image. Columns 2 through 4 represent the recovered solutions using periodic, reflective, and antireflective $\mathrm{BCs}$, respectively.

\subsection{Results}

The primary purpose of the next series of numerical examples is to compare the quality of the solutions provided by the Justen-Ramlau method using periodic BCs to solutions recovered under the assumption of reflective and antireflective BCs. Also of interest is the suitability of the Justen-Ramlau method under reflective and antireflective BCs as a truly blind deconvolution method. With these considerations in mind, a first set of test images were blurred, with no additive noise, using each of the three described PSF types. The images were subjected to blur at a mild or heavy intensity level, which was set by varying the parameter $\sigma$. Each of the resulting images was then restored by using a Gaussian PSF with the generalized Justen-Ramlau method. For each restoration, periodic, reflective, and antireflective boundary conditions were used for comparison. Numerical experimentation with the original Justen-Ramlau method led to the observation that the spread of exact PSF must not be underestimated by the estimated PSF. Following this observation, the estimated PSF $\bar{K}$ for each restoration was a Gaussian PSF with an overall spread similar to that of the exact PSF. Just as in the original work [13], an optimal value of the parameter $\gamma$ was determined for each restoration by maximizing the PSNR between the restored image and an extracted central portion of the corresponding exact image. 

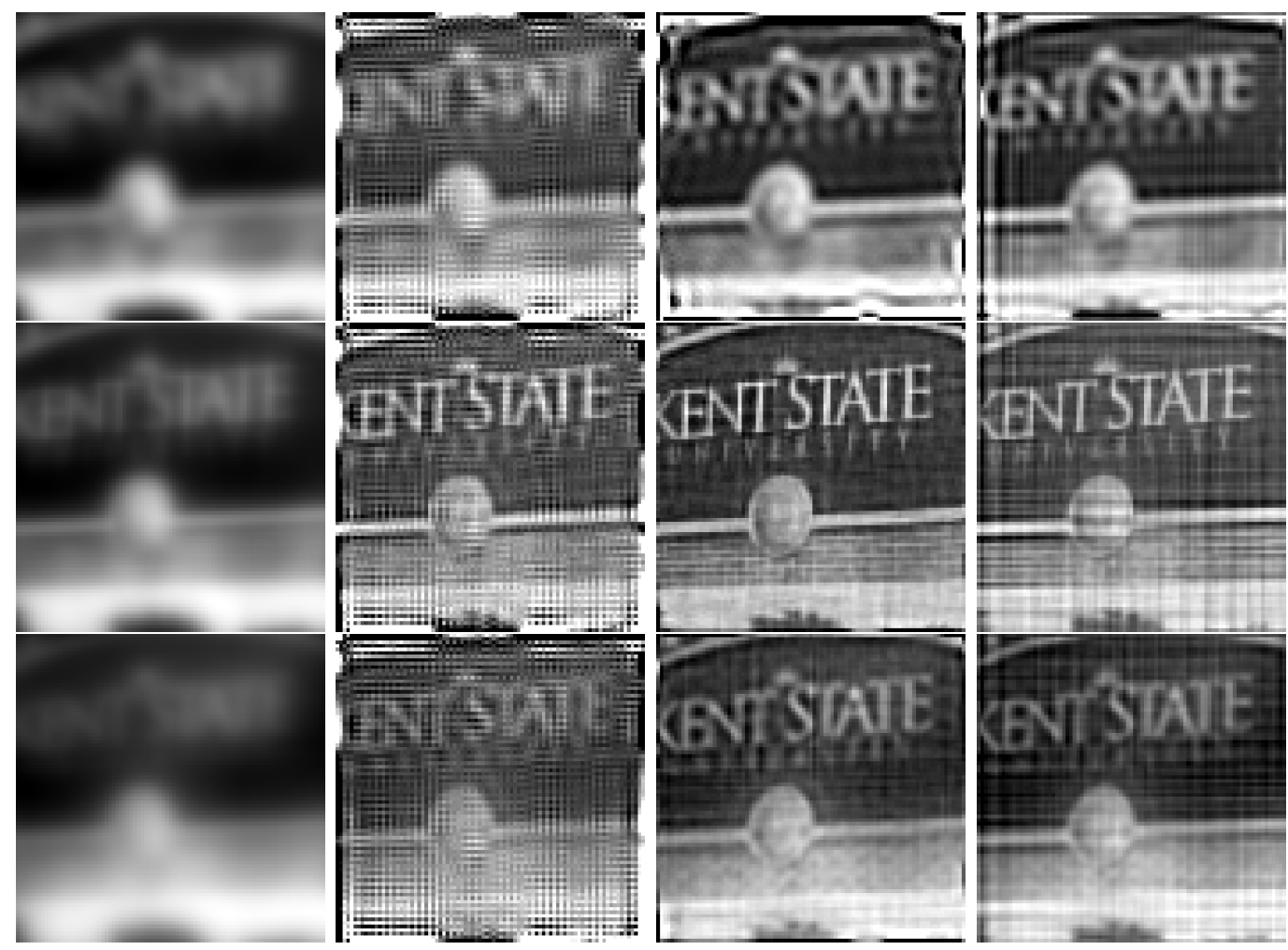

Figure 7: Kent image subjected to heavy blurring, and the corresponding restorations. Rows 1 through 3 represent blurred images constructed using Gaussian, Laplacian, and Cauchy PSFs, respectively. The first column of each row represents the blurred image. Columns 2 through 4 represent the recovered solutions using periodic, reflective, and antireflective BCs, respectively. 

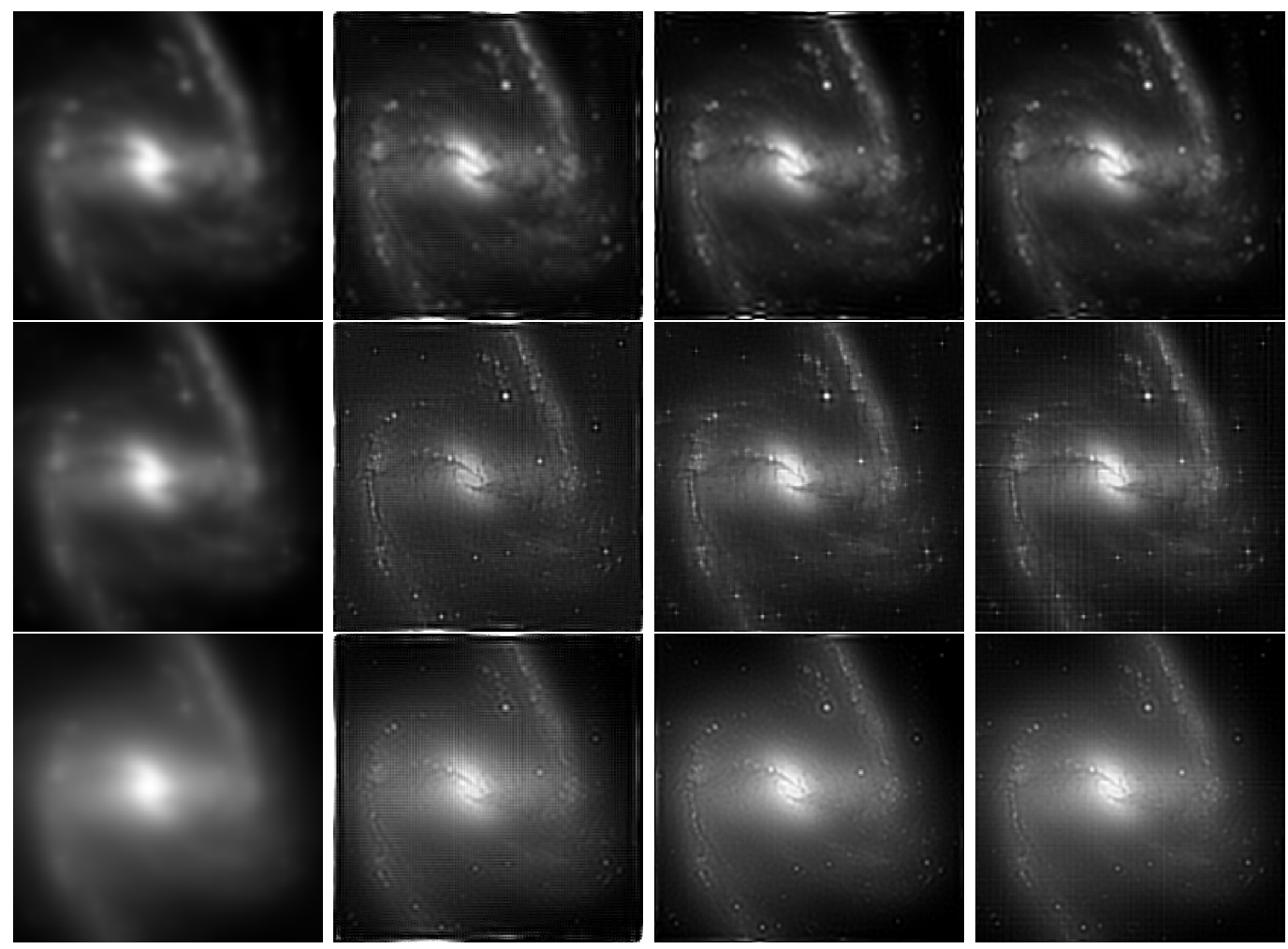

Figure 8: Galaxy image subjected to mild blurring, and the corresponding restorations. Rows 1 through 3 represent blurred images constructed using Gaussian, Laplacian, and Cauchy PSFs, respectively. The first column of each row represents the blurred image. Columns 2 through 4 represent the recovered solutions using periodic, reflective, and antireflective BCs, respectively. 

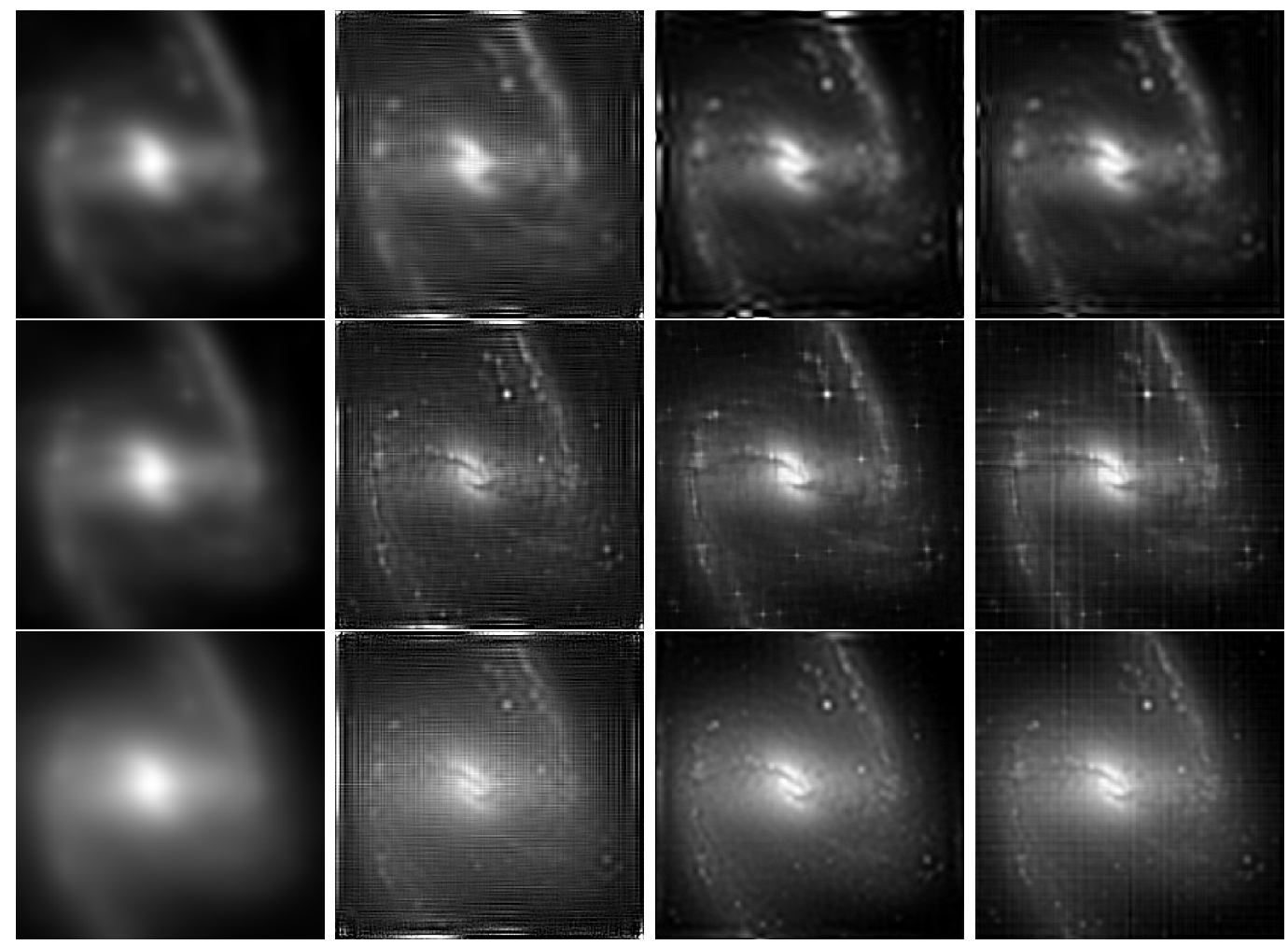

Figure 9: Galaxy image subjected to heavy blurring, and the corresponding restorations. Rows 1 through 3 represent blurred images constructed using Gaussian, Laplacian, and Cauchy PSFs, respectively. The first column of each row represents the blurred image. Columns 2 through 4 represent the recovered solutions using periodic, reflective, and antireflective BCs, respectively. 
In Figures 6 through 9, each row represents blurring according to a different type of PSF. The first image in each column shows the blurred image to be restored, and the columns which follows show the image recovered under (in order from left to right) periodic, reflective, and antireflective BCs. It is notable how the solutions recovered under periodic boundary conditions become overly contaminated with waveform artifacts as the blurring is intensified. In comparison, these artifacts are less prevalent in the solutions computed under reflective and antireflective BCs. For both mild and heavier blurring, it is also notable that the antireflective boundary conditions appear to be the best overall when the images have been blurred with a Gaussian and Cauchy PSF, but the solutions recovered under reflective BCs are far clearer with Laplacian blurring. The PSNR-value for each restoration is shown in Table 1. To keep the example section reasonably short, we do not show restored PSFs. Illustrations for periodic boundary conditions can be found in [13].

Next, a series of restorations were performed with blurry images with additive noise present. Gaussian PSFs were used in the construction of these images at a mild blurring intensity. Restorations at $1 \%$ and $3 \%$ relative noise levels were performed using periodic, reflective, and antireflective BCs. Due to noise, additional regularization is performed via a smoothing operation on $\hat{g}$, as described in [13]. This is done by replacing $\hat{g}$ by

$$
\widehat{R_{\alpha} g_{i, j}}=\frac{\hat{g}_{i, j}}{1+\alpha\left(1+\lambda_{i, j}^{2}\right)^{2}}, \quad 1 \leq i \leq m, \quad 1 \leq j \leq n,
$$

where the $\lambda_{i, j}$ are eigenvalues of the discrete Laplacian operator under the applied BCs. Figures 10 and 11 show the blurred and noisy images, along with the recovered solutions. In the case of blurred and noisy data, it is noted that the antireflective boundary conditions seem to perform better than periodic or reflective ones at low noise levels, while reflective BCs seem to perform better at higher noise levels. We note that the method does not perform as well for blurred images with higher noise levels. The PSNR-value for each restoration is displayed in Table 2.

\section{Discussion and Conclusion}

The numerical examples of the previous section demonstrate that the Justen-Ramlau method can be more effective when BCs other than periodic ones are used to restore a blurred image. With reflective and antireflective boundary conditions, the method is a viable non-iterative blind deconvolution method. The global shape of the exact PSF need not be known in order to obtain a quality reconstruction.

\section{References}

[1] A. Aricò, M. Donatelli, J. Nagy, and S. Serra-Capizzano, The anti-reflective transform and regularization by filtering, in Numerical Linear Algebra in Signals, Systems, and Control, eds. S. Bhattacharyya, R. Chan, P. Van Dooren, V. Olshevsky, and A. Routray, Lecture Notes in Electrical Engineering, Vol. 80, Springer, New York, 2012, pp. 1-21. 


\begin{tabular}{|c|c|c|c|c|}
\hline Image & Blur intensity & $\mathrm{PSF}$ & $\mathrm{BC}$ & PSNR \\
\hline \multirow{18}{*}{ KSU } & \multirow{9}{*}{ Mild $(\sigma=1.5)$} & \multirow{3}{*}{ Gaussian } & Periodic & 32.17 \\
\hline & & & Reflective & 32.54 . \\
\hline & & & Antireflective & 33.07 \\
\hline & & \multirow{3}{*}{ Laplacian } & Periodic & 32.42 \\
\hline & & & Reflective & 33.73 \\
\hline & & & Antireflective & 33.54 \\
\hline & & \multirow{3}{*}{ Cauchy } & Periodic & 31.73 \\
\hline & & & Reflective & 31.99 \\
\hline & & & Antireflective & 32.02 \\
\hline & \multirow{9}{*}{ Heavy $(\sigma=3)$} & \multirow{3}{*}{ Gaussian } & Periodic & 31.05 \\
\hline & & & Reflective & 31.60 \\
\hline & & & Antireflective & 32.22 \\
\hline & & \multirow{3}{*}{ Laplacian } & Periodic & 30.87 \\
\hline & & & Reflective & 31.90 \\
\hline & & & Antireflective & 31.51 \\
\hline & & \multirow{3}{*}{ Cauchy } & Periodic & 31.16 \\
\hline & & & Reflective & 31.83 \\
\hline & & & Antireflective & 31.87 \\
\hline \multirow{18}{*}{ Galaxy } & \multirow{9}{*}{ Mild $(\sigma=3)$} & \multirow{3}{*}{ Gaussian } & Periodic & 34.64 \\
\hline & & & Reflective & 35.40 \\
\hline & & & Antireflective & 37.12 \\
\hline & & \multirow{3}{*}{ Laplacian } & Periodic & 34.21 \\
\hline & & & Reflective & 37.21 \\
\hline & & & Antireflective & 36.96 \\
\hline & & \multirow{3}{*}{ Cauchy } & Periodic & 34.41 \\
\hline & & & Reflective & 35.05 \\
\hline & & & Antireflective & 35.6 \\
\hline & \multirow{9}{*}{ Heavy $(\sigma=5)$} & \multirow{3}{*}{ Gaussian } & Periodic & 33.91 \\
\hline & & & Reflective & 34.78 \\
\hline & & & Antireflective & 36.09 \\
\hline & & \multirow{3}{*}{ Laplacian } & Periodic & 34.07 \\
\hline & & & Reflective & 36.66 \\
\hline & & & Antireflective & 36.37 \\
\hline & & \multirow{3}{*}{ Cauchy } & Periodic & 34.04 \\
\hline & & & Reflective & 34.90 \\
\hline & & & Antireflective & 34.97 \\
\hline
\end{tabular}

Table 1: Tabulation of the PSNR for each restoration of the noise free test images. 

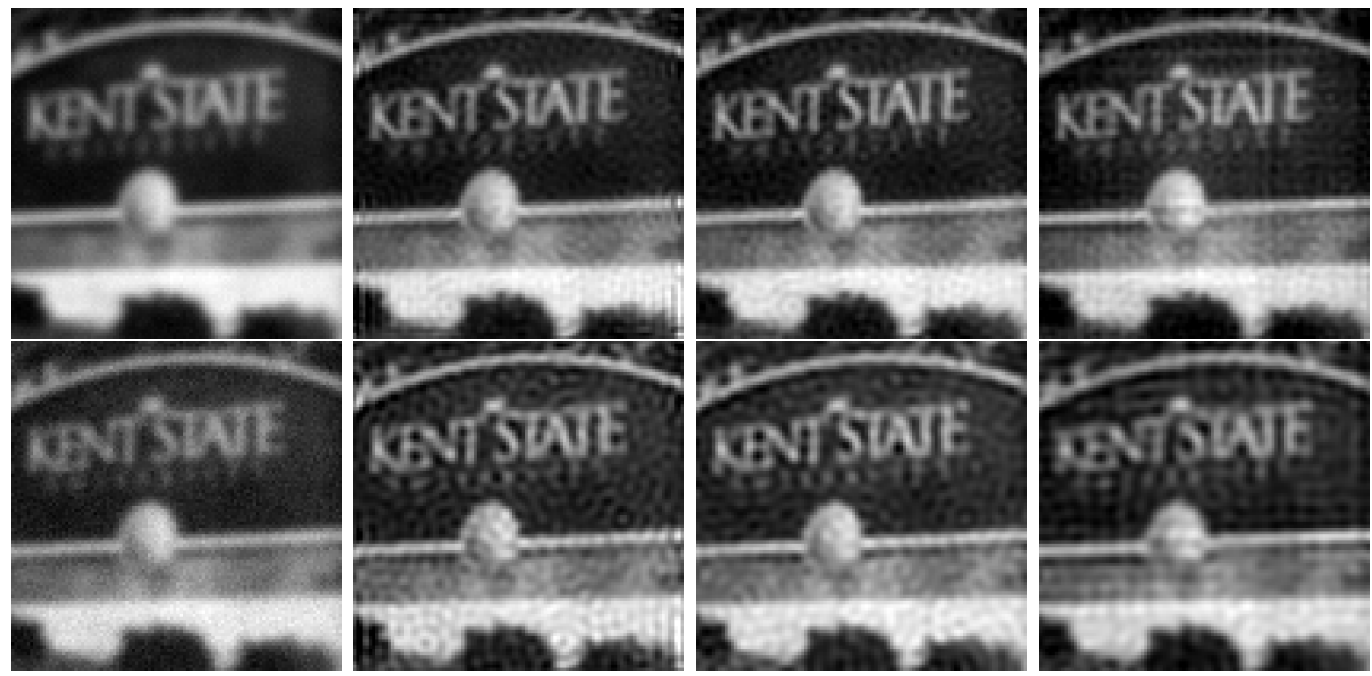

Figure 10: Kent image subjected to mild Gaussian blurring with noise, and the corresponding restorations. Rows 1 and 2 represent $1 \%$ and $3 \%$ relative noise, respectively. The first column of each row represents the blurred and noisy image. Columns 2 through 4 represent the recovered solutions using periodic, reflective, and antireflective BCs, respectively.
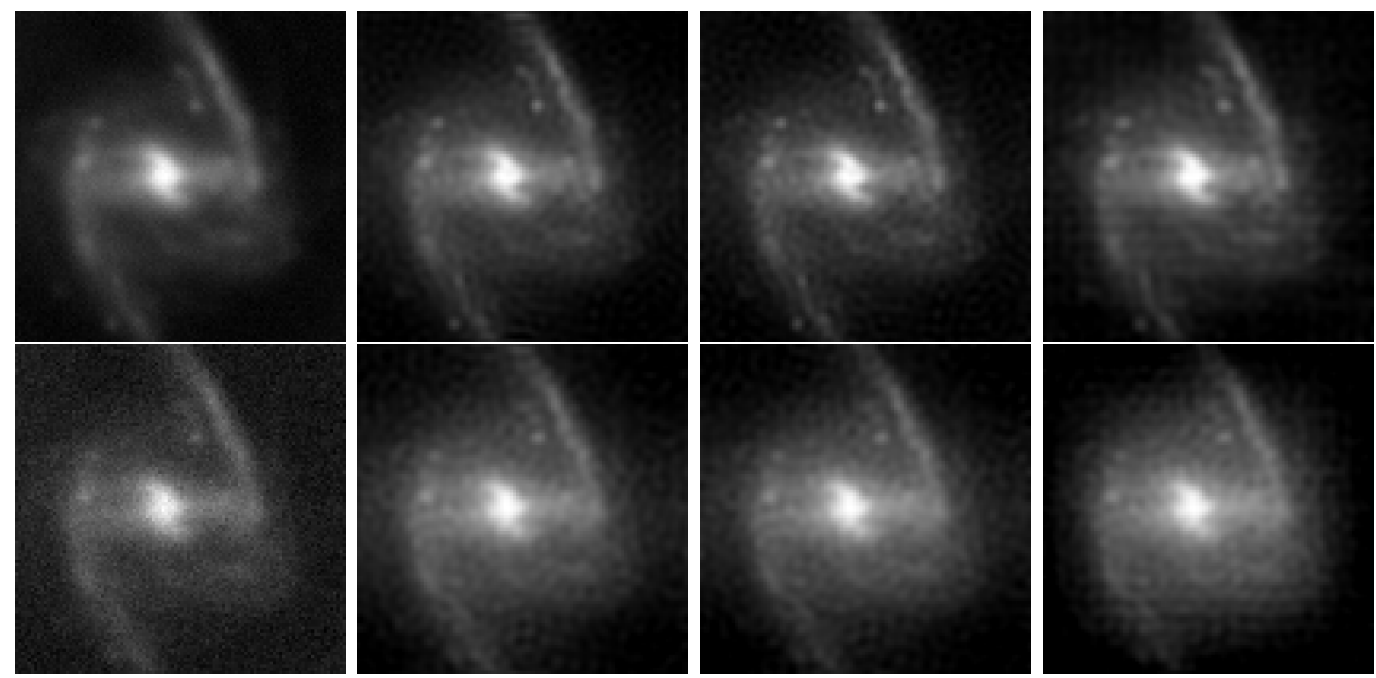

Figure 11: Galaxy image subjected to mild Gaussian blurring with noise, and the corresponding restorations. Rows 1 and 2 represent $1 \%$ and $3 \%$ relative noise, respectively. The first column of each row represents the blurred and noisy image. Columns 2 through 4 represent the recovered solutions using periodic, reflective and antireflective BCs, respectively. 


\begin{tabular}{|c|c|c|c|c|}
\hline Image & Blur intensity & PSF & $\mathrm{BC}$ & PSNR \\
\hline \multirow{6}{*}{$\mathrm{KSU}$} & \multirow{6}{*}{ Mild $(\sigma=1.5)$} & \multirow{3}{*}{$1 \%$ noise } & Periodic & 29.29 \\
\hline & & & Reflective & 30.13 \\
\hline & & & Antireflective & 31.49 \\
\hline & & \multirow{3}{*}{$3 \%$ noise } & Periodic & 32.60 \\
\hline & & & Reflective & 32.56 \\
\hline & & & Antireflective & 32.70 \\
\hline \multirow{6}{*}{ Galaxy } & \multirow{6}{*}{ Mild $(\sigma=3)$} & \multirow{3}{*}{$1 \%$ noise } & Periodic & 28.87 \\
\hline & & & Reflective & 31.20 \\
\hline & & & Antireflective & 30.26 \\
\hline & & \multirow{3}{*}{$3 \%$ noise } & Periodic & 27.95 \\
\hline & & & Reflective & 28.42 \\
\hline & & & Antireflective & 28.06 \\
\hline
\end{tabular}

Table 2: Tabulation of the PSNR for each restoration of the noisy images.

[2] G. R. Ayers and J. C. Dainty, Iterative blind deconvolution method and its application, Opt. Lett., 13 (1988), pp. 547-549.

[3] P. Campisi and K. Egiazarian, eds., Blind Deconvolution: Theory and Applications, CRC Press, 2007.

[4] A. S. Carasso, False characteristic functions and other pathologies in variational blind deconvolution. A method of recovery, SIAM J. Appl. Math., 70 (2009), pp. 1097-1119.

[5] T. F. Chan and C. K. Wong, Total variation blind deconvolution, IEEE Trans. Image Processing, 7 (1998), pp. 370-375.

[6] T. F. Chan and C. K. Wong, Convergence of the alternating minimization algorithm for blind deconvolution, Linear Algebra Appl., 316, (2000), pp. 259285.

[7] Y.-W. Fan and J. G. Nagy, An efficient computational approach for multiframe blind deconvolution, J. Comput. Appl. Math., 236 (2012), pp. 2112-2125.

[8] P. C. Hansen, Rank-Deficient and Discrete Ill-Posed Problems: Numerical Aspects of Linear Inversion, SIAM, Philadelphia, 1988.

[9] P. C. Hansen, J. G. Nagy, and D. P. O'Leary, Deblurring Images: Matrices, Spectra, and Filtering, SIAM, Philadelphia, 2006.

[10] L. He, A. Marquina, and S. J. Osher, Blind deconvolution using TV regularization and Bregman iteration, Int. J. Imaging Syst. Technol., 15 (2005), pp. $74-83$.

[11] R. A. Horn and C. R. Johnson, Topics in Matrix Analysis, Cambridge University Press, Cambridge, 1991. 
[12] H. Ji, J. Li, Z. Shen, and K. Wang, Image deconvolution using a characterization of sharp images in wavelet domain, Appl. Comput. Harm. Anal., 32 (2012), pp. 295-304.

[13] L. Justen and R. Ramlau, A non-iterative regularization approach to blind deconvolution, Inverse Problems, 22 (2006), pp. 771-800.

[14] A. Levin, Y. Weiss, F. Durand, and W. T. Freeman, Understanding and evaluating blind deconvolution algorithms, Proc. IEEE Conference on Computer Vision and Pattern Recognition, CVPR, IEEE, 2009, pp. 1964-1971.

[15] B. H. Liao and M. Ng, Blind deconvolution using generalized cross validation approach to regularization parameter estimation, IEEE Trans. Image Processing, 21 (2012), pp. 562-572.

[16] R. Molina, J. Mateos, and A. K. Katsaggelo, Blind deconvolution using a variational approach to parameter, image, and blur estimation, IEEE Trans. Image Processing, 15 (2006), pp. 3715-3727.

[17] M. Ng, R. Chan, and W.-C. Tang, A fast algorithm for deblurring models with Neumann boundary conditions, SIAM J. Sci. Comput., 21 (1999), pp. 851-866.

[18] M. K. Ng and R. Plemmons, Blind deconvolution and structured matrix computations with applications to array imaging, in Blind Deconvolution: Theory and Applications, eds. P. Campisi and K. Egiazarian, CRC Press, 2007, pp. $377-418$.

[19] M. K. Ng, R. Plemmons, and S. Qiao, Regularization of RIF blind image deconvolution, IEEE Trans. Image Process., 9 (2000), pp. 1130-1134.

[20] S. Serra-Capizzano, A note on antireflective boundary conditions and fast deblurring models, SIAM J. Sci. Comput., 25 (2003), pp. 1307-1325.

[21] G. Spaletta and L. Caucci, Constrained iterations for blind deconvolution and convexity issues, J. Comput. Appl. Math., 197 (2006), pp. 29-43. 